\title{
Lymphoproliferative disorder in the context of hypereosinophilic syndrome
}

Cristiano Claudino Oliveira ${ }^{*}$, Luan Kucko ${ }^{2}$, Eric Jun Hirama ${ }^{2}$, Helena Maciel Guerra ${ }^{2}$, Lied Martins Santiago $^{3}$, Manuella Pacífico Segredo ${ }^{3}$, Ligia Niero-Melo ${ }^{4}$ and Maria Aparecida Custódio Domingues ${ }^{5}$

${ }^{1}$ Department of Pathology, Botucatu School of Medicine, São Paulo State University, Botucatu, São Paulo, Brazil

${ }^{2}$ Academic of medicine, Botucatu School of Medicine, São Paulo State University, Botucatu, São Paulo, Brazil

${ }^{3}$ Department of Pediatrics, Botucatu School of Medicine, São Paulo State University, Botucatu, São Paulo, Brazil

${ }^{4}$ Department of Internal Medicine, Botucatu School of Medicine, São Paulo State University, Botucatu, São Paulo, Brazil

${ }^{5}$ Department of Pathology, Botucatu School of Medicine, São Paulo State University, Botucatu, São Paulo, Brazil

\begin{abstract}
Hypereosinophilic Syndrome (HES) is a condition related to helminthiasis, allergies, vasculitis, adverse reactions to drugs or malignant neoplasms. We report a case of a boy, 7 years old, presenting abdominal pain, vomiting, tachidispnea and leukocytosis with predominance of eosinophils. Bone marrow biopsy revealed intense granulocytic hypercellularity characterized by eosinophilia, as well as immature lymphoid cells in the interstitium, associated with trabecular bone infiltration. The immunohistochemical study revealed positivity for CD79a and TDT. Peripheral blood flow cytometry demonstrated 6.5\% of lymphoid blasts. The diagnosis was Lymphoproliferative Disease associated with Hypereosinophilia: Acute Lymphoblastic Leukemia. The association presented is infrequent and its aggressiveness is determined by the physiological limitations imposed by the damages secondary to eosinophilia.
\end{abstract}

\section{Introduction}

Hypereosinophilic syndrome (HES) is characterized by heart and central nervous system damage due to eosinophil products. HES diagnosis depends on peripheral blood and clinical features. Bone marrow is important in excluding hematopoietic malignances, as leukemia and lymphoma [1]. We report a rare case about a child with clinical presentation of HES whose bone marrow analysis indicated a diagnosis of leukemia.

\section{Case report}

Male child, 7 years old, presenting abdominal pain and vomiting for four days. Physical examination revealed severe tachydyspnea, without clinical infection signals. The blood count showed leukocytosis (194,000 cells $/ \mathrm{mm}^{3}$, reference value: $4,0-11,0$ cells $\left./ \mathrm{mm}^{3}\right)$ with a predominance of eosinophils ( $64 \%$ or 124,160 cells $/ \mathrm{mm}^{3}$, reference value: $2,0-4,0 \%)$. Given these findings, the diagnosis of congestive heart failure, in the context of hyperosinophilia, was considered and the echocardiogram was requested. This exam showed segmental contractile alterations with preserved ejection fraction, associated with signs of mild/moderate pulmonary arterial hypertension.

In order to exclude hematopoietic malignancies, the patient was submitted a bone marrow biopsy and a bone marrow aspirate with morphological and immunophenotype evaluated by immunohistochemistry. Peripheral blood sample was also evaluated by flow cytometry.

Bone marrow biopsy revealed preserved global cellularity for age. There was intense hypercellularity of granulocytic series with maturative delay characterized by intermediate eosinophilic precursors and mature eosinophils in a lower proportion (Figures 1A and 1B). Hypercellularity of the lymphocytic series with maturation delay with predominance of immature cells in the interstitium was also noted (Figure 1C, 1D and 1E). The trabecular bone exhibited foci of infiltration.

Peripheral blood flow cytometric examination demonstrated $6.5 \%$ of lymphoid blasts. The immunohistochemical study revealed positivity for CD79a (Figure $1 \mathrm{~F}$ ) and TDT (Figure $1 \mathrm{G}$ ) in $2 \%$ and $10 \%$ of immature cells, respectively. There was extensive positivity for myeloperoxidase due to the hypercellularity of the granulocytic series at the expense of eosinophilic precursors (Figure $1 \mathrm{H}$ ). The clinical, flow cytometric and immunohistochemical data indicated the diagnosis of Lymphoproliferative Disease associated with Hypereosinophilia: Acute Lymphoblastic Leukemia, with B immunophenotype (B-ALL).

The patient was treated with standard chemotherapy for these cases (BFM-2002, Berlin-Frankfurt Muster European Group for ALL treatment) and leukopheresis, evolving with several intercurrences secondary to immunosuppression and, especially, cardiac repercussions secondary to eosinophilia.

Correspondence to: Cristiano Claudino Oliveira, Departamento de Patologia, Faculdade de Medicina de Botucatu, Universidade Estadual Paulista (FMB UNESP), Botucatu, São Paulo, Brazil; E-mail: cristiano_c_oliveira@hotmail.com

Key words: precursor cell lymphoblastic leukemia-lymphoma, eosinophilia, hypereosinophilic syndrome, immunohistochemistry

Received: August 07, 2017; Accepted: August 17, 2017; Published: August 21, 2017 


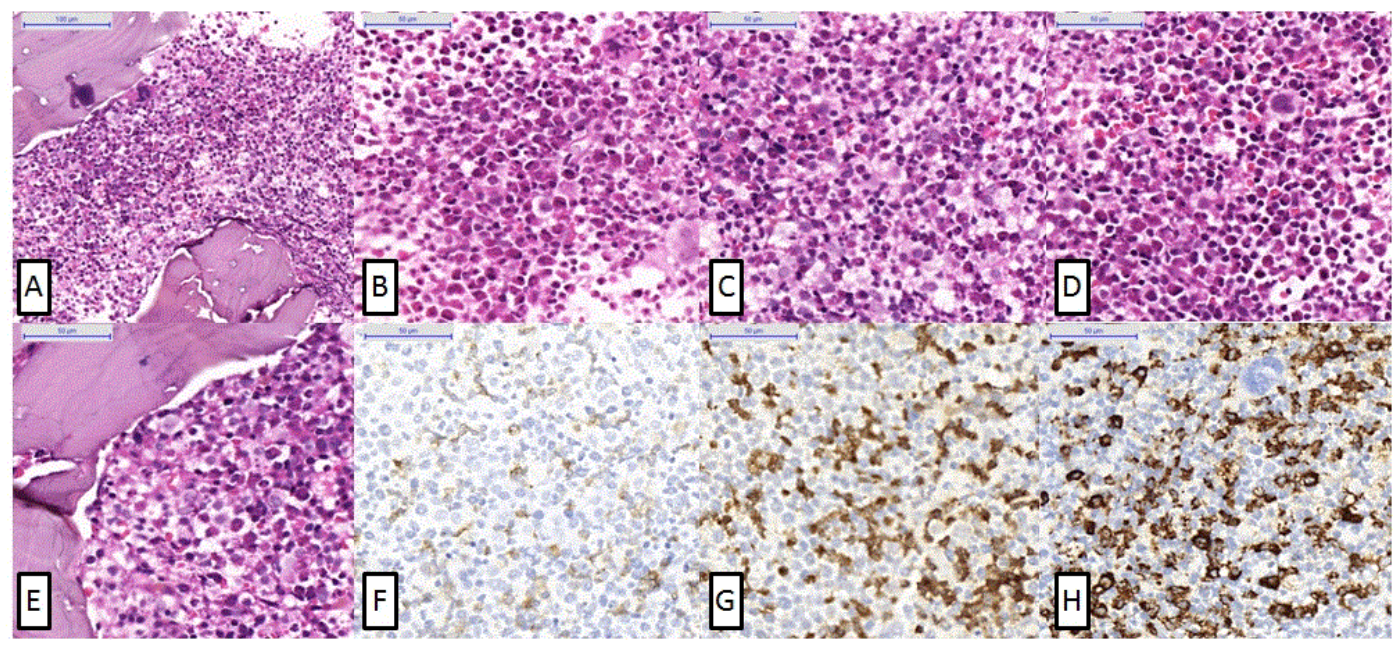

Figure 1. Figure 1-A (200x, H\&E) and B (400x, H\&E) show an intense hypercellularity of granulocytic series with maturative delay characterized by intermediate eosinophilic precursors and mature eosinophils in a lower proportion. C (400x, H\&E), D (400x, H\&E) and E (200x, H\&E) show Hypercellularity of the lymphocytic series with maturation delay with predominance of immature cells in the interstitium. F (400x, CD79a) shows B-cells. In that lymphocytosis, there is almost $2 \%$ of B-cells. G (400x, TDT) confirms the immature cells. They are immature neoplastic B-cells. H (400x, myeloperoxidase) confirms that these neoplastic B-cells are among granulocytic cells which stain for myeloperoxidase.

\section{Discussion}

Eosinophilia is a condition that may be related to morbidities such as helminthiasis, allergies, vasculitis, adverse drug reactions and malignant neoplasms [1,2]. HES occurs when there is peripheral eosinophilia greater than $1.5 \times 10^{9} / \mathrm{L}$ or more associated to an organ damage mediated by increased numbers of eosinophils. Hypereosinophilia is defined as the simple increase in peripheral blood eosinophil count greater than $1.5 \times 10^{9} / \mathrm{L}^{1}$.

HES is a rare disease that occurs with sustained eosinophilia in the peripheral blood or bone marrow. The disease predominantly affects males (nine males for one woman), in the age group of 20 to 50 years [2]. Less commonly, the disease affects individuals at the extreme age [2,3]. Clinically, patients with HES may present dyspnea, weakness, cough, myalgias, fever and cutaneous rash. The common laboratory finding is

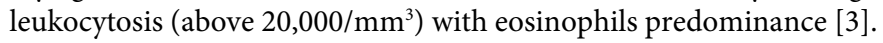

Tissue infiltration by eosinophils is the promoter of major organ damage due to the release of cell granules [2]. The systemic effects of sustained eosinophilia include cutaneous, pulmonary, gastrointestinal and, mainly, cardiac changes. Approximately $20 \%$ of HES patients had cardiac damages not related to other comorbidities, and $6.0 \%$ of the subjects presented them at the first moment of the disease. Cardiac damage, as well as systemic damage, is caused by infiltration of eosinophils into cardiac fibers with degranulation of toxic mediators to tissues. Endocardial changes, restrictive cardiomyopathies and valvular insufficiencies are examples of cardiac repercussions of HES [3]. On admission, our patient exhibited cardiac alterations, which are confirmed, later, by echocardiogram.

The occurrence of hypereosinophilia and ALL is rare, with incidence less than $1.0 \%$. Review of the literature by Rappanoti [2] et al. published in 2010 raised 52 cases of the association reported in 44 articles. The clinical importance of the association is with regard to the treatment, since hypereosinophilia promotes cardiac changes that limit the use of certain drugs and restrict the clinical evolution of the patients [2].

Approximately $10 \%$ of HES patients present cytogenetic alteration of FIP1L1-PDGFRA fusion [3-5]. In the case of patients with HES and ALL, in more than half of the cases reported in the literature, there is the detection of genetic abnormalities, with the $t(5 ; 14)(\mathrm{q} 31 ; \mathrm{q} 32)$ translocation being the most commonly diagnosed. It is interesting to note that this translocation, in more than $90 \%$ of cases, is detected only in lymphoid blasts and not in eosinophils. These data support the theory that eosinophilia would be a reactive phenomenon, secondarily induced by the elevated expression of cytokine levels, such as interleukin 5, interleukin 3 and granulocyte stimulating factor, released by leukemic cells [4-7].

ALL in the context of Hypereosinophilia is a rare subtype of malignant lymphoproliferative disease whose aggressiveness is determined by the physiological limitations imposed by secondary damage to eosinophilia [5]. In the present case, it is noted that the definitive diagnosis was achieved by the correlation of morphological data with immunophenotype aspects. The patient death is associated to his cardiac repercussions secondary to eosinophilia.

\section{References}

1. Tefferi A, Gotlib J, Pardanani A (2010) Hypereosinophilic Syndrome and Clonal Eosinophilia: Point-of-Care Diagnostic Algorithm and Treatment Update. Mayo Clin Proc 85:158-164 [Crossref]

2. Rapanotti MC, Caruso R, Ammatuna E, Zaza S, Trotta L, et al. (2010) Molecular characterization of paediatric idiopathic Hypereosinophilia. Br J Haematol 151:440446. [Crossref]

3. Gotlib J. World Health Organization-defined eosinophilic disorders: 2011 update on diagnosis, risk stratification, and management. Am J Hematol 86:678-688.

4. Nie YL, Jan SL, Fu LS, Chang TK, Wang JD (2010) Congestive heart failure as presentation of acute lymphoblastic leukaemia with eosinophilia. Br J Haematol 149: 633. [Crossref]

5. Sutton R, Lonergan M, Tapp H, Venn NC, Haber M, et al. (2008) Two cases of hypereosinophilia and high-risk acute lymphoblastic leukemia. Leukemia 22: 14631465. [Crossref]

6. Reiter A, Gotlib J2 (2017) Myeloid neoplasms with eosinophilia. Blood 129: 704-714 [Crossref]

7. Gotib J. World Health Organization-defined eosinophilic disorders: 2015 update on diagnosis, risk stratification, and management. Am J Hematol 90:1078-1089.

Copyright: (C2017 Oliveira CC. This is an open-access article distributed under the terms of the Creative Commons Attribution License, which permits unrestricted use, distribution, and reproduction in any medium, provided the original author and source are credited. 